\title{
Study of Quantum Dots Labeled Trypanosoma cruzi - Rhodnius prolixus Interaction by Real Time Confocal Images
}

\author{
A. A. de Thomaz*, D. B. Almeida*, A. Fontes**, C. V. Stahl***, J. R. Santos- \\ Mallet***, S. A.O. Gomes***, D. Feder**** and C. L. Cesar* \\ *Laboratório de Aplicações Biomédicas de Lasers, Instituto de Física “Gleb Wataghin”, \\ UNICAMP, Campinas, SP, Brazil \\ **Depto de Biofísica e Radiobiologia, CCB, UFPE, Recife, PE, Brazil \\ ***Laboratório de Transmissores de Leishmanioses, Setor de Morfologia, \\ Ultraestrutura e Bioquímica de Artrópodes e Parasitos, IOC-FIOCRUZ-Rio de Janeiro, \\ RJ, Brazil; \\ ****Laboratório de Biologia de Insetos, GBG, Universidade Federal Fluminense-UFF, \\ Rio de Janeiro, RJ, Brazil;
}

Semiconductor quantum dots (QDs) are highly fluorescent nanocrystals markers that allow long term live biological processes observations because they do not present photobleaching and do not destroy the parasites. In this paper we show that fluorescent core shell quantum dots can be used to perform studies of live parasite-vector interaction processes without any observable vitality effect on the parasites. These nanocrystals were synthezised in our own facilities in aquous medium and physiological $\mathrm{pH}$, which is very important for monitoring live cells activities, and conjugated with molecules such as lectins to label specific carbohydrates involved on the parasite-vector interaction. These QDs allowed us to acquire real time confocal images sequences of live T. cruzi $-R$. prolixus interactions for an extended period, causing no damage in the cells. By zooming to the region of interest we have been able to acquire confocal images at 3 to 4 frames per second rate.

QDs labeling has not affected the development of T. cruzi cells. Parasite cells labeled with yellow-emitting CdSe QDs were observed in confocal microscopy after 3 days, showing that the growing and the cell division had not been altered, as seen in figure 1. In vitro experiments showed QDs labeled posterior midgut epithelial cells of $R$. prolixus with living T. cruzi, also labeled, attached to PMM as shown in figure 2. Figure 3 shows vesicles containing QDs bioconjugated with SNA that have specificity to galactose (gal) and $\mathrm{N}$-acetylgalactosamine (galNac). This result indicates that these QDs were internalized by endocitosis. When endocitocis was blocked by incubation at $4{ }^{\circ} \mathrm{C}$ (not shown), parasites cells were not labeled by QDs even after extended incubation ( $>2 \mathrm{hs}$ ) comparing with parasites incubated with QDs at room temperature, where we could observe a high QDs label.

In summary, our results showed that it is possible to use physiological fluorescent markers to label alive parasites and cells insect vector. Our results also show that they can be functionalized with lectins to specifically mark surface carbohydrates on perimicrovillar membrane of $R$. prolixus to follow, visualize and understand, interaction between vectors and its parasites in real time. 


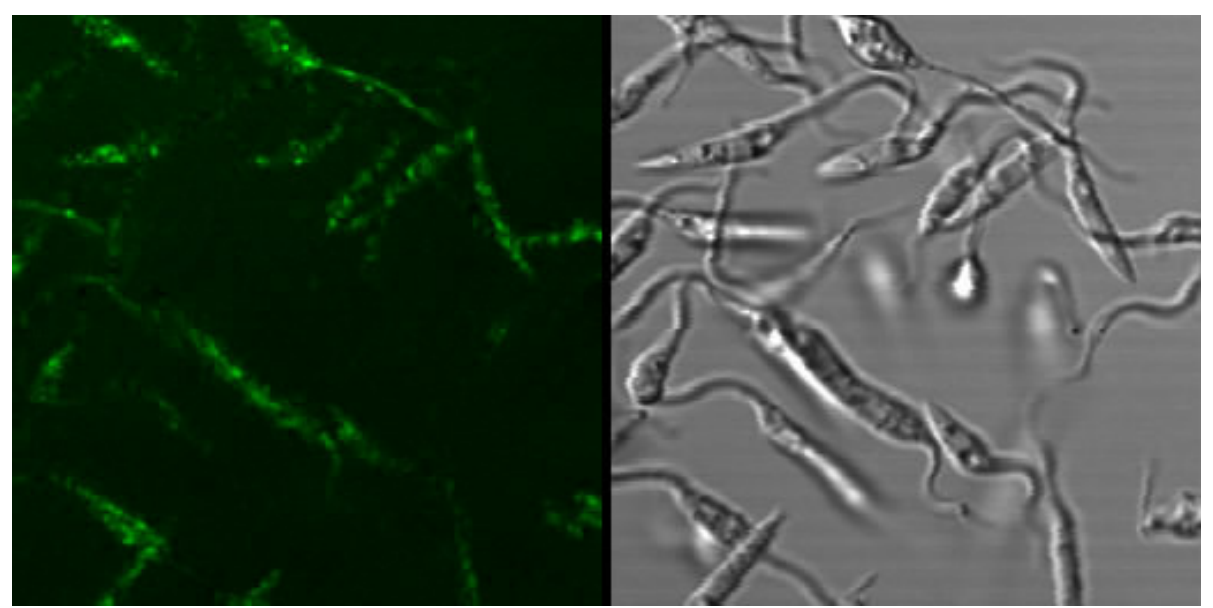

Fig 1 Live parasite cells imaging - Left- In vitro incubation of Trypanosoma cruzi cells with yellow emitting CdSe quantum dots and shows binary division of parasites. RightLaser transmission

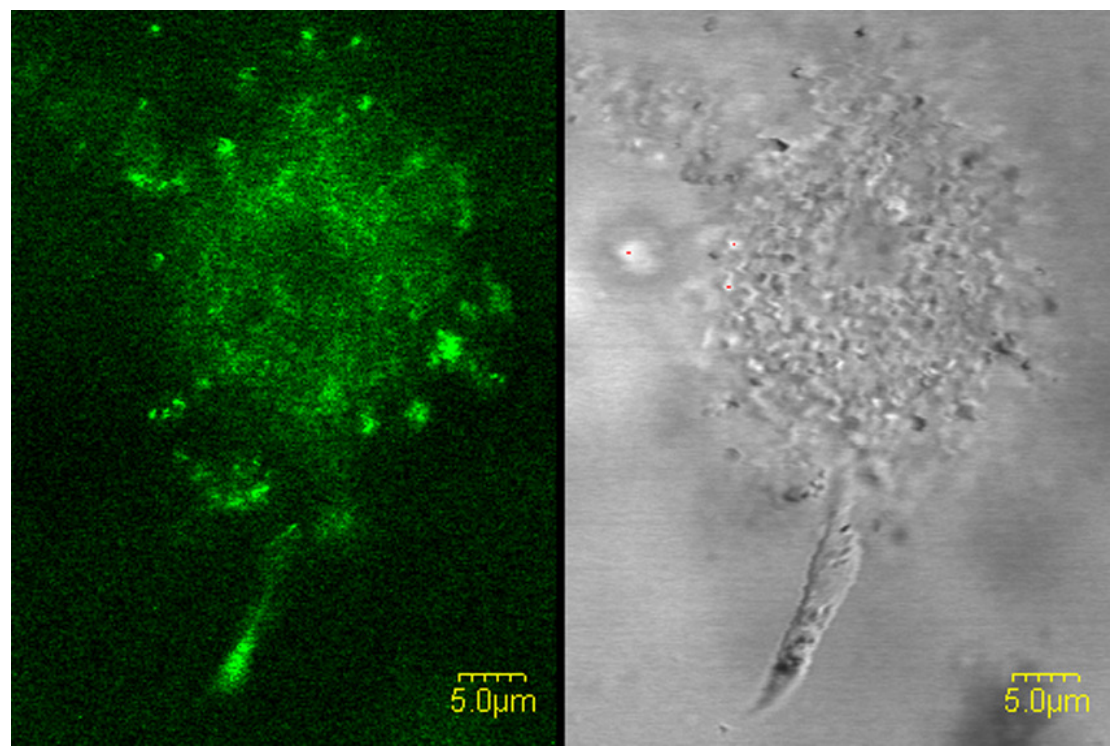

Fig 2 Image of $T$. cruzi adhered to the intestine of $R$. prolix. Left - Fluorescence of QDs. Right - Laser transmission

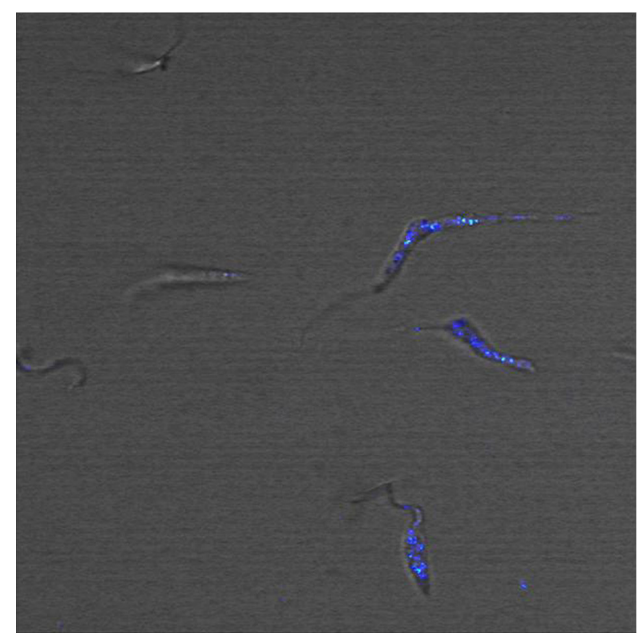

Fig 3 Live parasite cells imaging using quantum dots bioconjugates with lectin (Sambacus Agglutinin Nigra - SNA) 